\title{
First Waste Plastic Conversion into Liquid Fuel by Using Muffle Furnace through Reactor
}

\author{
Moinuddin Sarker*, Mohammad Mamunor Ras hid, Mohammed Molla
}

Natural State Research, Inc. Department of Research and Development, 37 Brown House Road (2 ${ }^{\text {nd }}$ Floor), Stamford, CT-06902, USA

\begin{abstract}
The oil consumption in the United States and Canada is much higher per capita compared to rest of the world. Even though only $37.1 \%$ of oil is used as a supply source for all the major demand sectors like, industrial transportation, residential and electric power, it is a limited natural resource that cannot be replaced. Many alternative sources such as, solar, hydro power, wind and some others are available in s mall numbers. In the current economic situation of the world, it is practically impossible to bring these high cost energy sources into mass numbers. A method to convert waste plastics into liquid fuel has been implemented and exercised in a closed muffle furnace and a stainless steel reactor unit. The process involves thermally breaking down the hydrocarbon bonds of polymers in a muffle furnace with temperature of $420^{\circ} \mathrm{C}$ to turn into liquid slurry and that slurry is then processed through a reactor with te mperature of $380^{\circ} \mathrm{C}$ to convert into liquid fuel. The fuel obtained is of quality grade and has been tested to work with most conventional engines, generators and such. This technology main point is to reduce the amount of carbon footprint caused by abundant waste plastic. This present technology will remove waste plastics that are harmful to our environment and convert those harmful waste plastics in to valuable oil sources to full fill the energy crisis and strengthen the economy.
\end{abstract}

Keywo rds Waste Plastic, Liquid Fuel, Hydrocarbon, Conversion, Thermal Process, Muffle Furnace

\section{Introduction}

Energy crisis and environmental degradation by polymer wastes have been imperative to find and propose technologies for recovery of raw materials and energy from non-conventional sources like organic wastes, plastic wastes, scrap tires, etc. A variety of methods and processes connected with global or national policies have been proposed worldwide. A new type of a tubular reactor with the molten metal bed is proposed for conversion of waste plastics to fuel like mixture of hydrocarbons. The results of the thermal degradation of polyolefins in the laboratory scale set-up based on this reactor are presented in the paper. The melting and cracking processes were carried out at the temperature $300-420^{\circ} \mathrm{C}$. The final product cons isted of gaseous product $6 \%$ and liquid product $85 \%$ stream. Few amounts of residue products were produced that $6 \%$. The light, " "gasoline" fraction of the liquid hydrocarbons mixtu re is $\left(\mathrm{C}_{4}-\mathrm{C}_{11}\right)$ made the liquid product. It may by used for fuel production or electric ity generation[1]. Since the continuous increase in polymer production and consumption leads to accumulation of large amounts of plastic wastes that pose serious environmental problems, the conversion of these

* Corresponding author:

msarker@naturalstat eres earch.com (Moinuddin Sarker)

Published online at http://journal.sapub.org/ijee

Copyright (C) 2012 Scientific \& Academic Publishing. All Rights Reserved wastes into a common fuel oil can be considered as their most promising recycling method. So me polymer materials e.g., polystyrene (PS), can be decomposed thermally in high yields to the monomers. However, this is not true for polyethylene (PE) or polypropylene (PP), which is among the most abundant polymeric waste materials, typically making up 60-70\% of mun icipal solid waste. However, most studies have mainly concentrated on the catalytic degradation of pure polymers. It would be desirable to convert these waste polyolefins into products of value other than the monomers, because they offset the collection and pyrolysis costs. It seems also that in the process of plastic pyrolysis, a particular kind of catalyst is effective for a particular kind of plastic. Unfortunately it can only be applied to pure plastics and is not recommended for mixed plastic wastes. A more difficult task is tertiary recycling of commingled post-consumer plastic waste since it consists of not only hydrocarbons but also nitrogen and sulphur containing mixed polymers as well as some modified materials. Study of the product distributions from the degradation of mixture of post-commercial polymer waste (LDPE/HDPE/PP/PS) and to especially develop a suitable reaction model for achieving post use polymer is recycling[2]. In view of their versatility and relatively low cost, the consumption of plastic materials has been growing steadily, although the disposal of waste plastics constitutes a severe environmental problem due mainly to their chemical inertness. While a polymer recycling is a requirement to 
mitigate their impact on the environment[3], various tertiary recycling processes are attractive, since they produce valuable chemicals or fuels [4, 5]. Considering polyolefins, polyethylene and polypropylene have a massive production and consumption in a large number of applications. The tertiary recycling of polyolefins (particularly polyethylene) has been attempted under different approaches. The most used laboratory technique with some variations, is that of contacting the plastic with the catalyst in a closed environment, heating them together until reaction temperature, and allowing for a certain reaction-time; products are then separated and analyzed (e.g.[6,7]). However the process we use doesn't require the use of any catalyst therefore the process is more cost effective and environmentally friendly compared to the ones that are studied in previous research. Thermal or catalytic cracking of waste plastics is one of the possible methods of their utilization[3-10]. As a result of the cracking at $400 \mathrm{C}$ or higher process temperature some quantities of hydrocarbon mixtures in the form of gas, liquid products (gasoline and diesel fuel boiling range) as well as higher boiling liquid residue or solid can be obtained[3,8,10]. All these products can be used as fuels or fuel components. Especially liquid products of gasoline and diesel fuel boiling range can be applied as components of engine fuels. It is however necessary to reme mber that products of cracking or pyrolysis of polyolefin's are highly unsaturated and therefore they have to be further submitted to hydrogenation and skeletal isomerization if they are to be applied as engine fuels. Application is cracking or hydro cracking catalyst and higher process. Temperature can enlarge conversion of waste plastics. The main goal of application of hydro cracking catalyst and hydrogen is hydro cracking of plastics and hydrogenation of olefins in process products. Taking into account these premises in these studies waste samples waste plastics were used as raw materials in thermal process by using muffle furnace through reactor[8-15].

\section{Materials and Method}

\subsection{Materials}

Waste plastic raw materials are collected from nearby groceries and supermarkets. After receiving the waste plastics they are separated out of all foreign materials such as food particle, paper, dust, cloth, glass, metal etc. The plastics are then washed with liquid soap ( $7^{\text {th }}$ generation company) and after washing they are dried for several hour in room temperature. After they are completely dried the plastics are cut into small pieces with scissor to size 3-4 mm. During waste plastic washing period waste water was generated and waste water was kept into separate container for waste water treatment purpose. Waste water is treated with alkali and acidic method. Alkali is $\mathrm{NaOH}$ and acid ic solution is Potash Alum with different normality.

\subsection{Raw Materials Pre Analysis}

Before liquefaction process the raw plastic are analyzed using Gas Chro matography and Mass Spectrometer (GC/MS) with Pyroprobe (CDS) gasification system and gasification system temperature was use $1200^{\circ} \mathrm{C}$, FT-IR (Spectrum 100) ATR system, TGA (Pyris-1) and EA-2400. GC/MS is used to identify the compound structures of the plastics. This would give a clear idea of how the mo lecules of the poly mers will react in the temperature that will be used during the liquefaction process FT-IR is used to identify the functional group of the plastics. TGA was used to find the onset temperature measurement telling what temperature range needs to be used to melt the plastic during the liquefaction process and finally the elemental analyzer (EA-2400) was used to identify the carbon, hydrogen, nitrogen percentage of the plastics. Waste plastic also per-analy zed by ICP (ASTM D1976) and found substantial metal content are percent such as Silver, Aluminum, Boron, Barium, Calcium, Chromium, Copper, Iron, Potassium, Lithium, Magnesium, Molybdenum, Sodiu m, Nickel, Phosphorus, Lead, Antimony, Silicon, Tin, Strontium, Titanium, Thallium, Vanadium and Zinc etc. all metal content present limit inside waste plastic ppm level. That metal content of the waste plastics act as a catalyst during the fuel production process. The metals are added to plastics during their production period to strengthen them as additive for color, hardness, softness and other good shape and additives was used 1-3\%.

\subsection{Experimental Process Description}

A muffle furnace type F 6000 from Barnstead International Company was initially used to convert the plastic samples into liquid slurry. The plastics were placed into a ceramic crucible and covered. Ceramic crucible can tolerate temperature up to $1200{ }^{\circ} \mathrm{C}$ and muffle furnace temperature can go up to $1400^{\circ} \mathrm{C}$. The experiment took place inside a vacuumed fume hood. The crucible with $300 \mathrm{gm}$ of plastics was placed inside the muffle furnace then the door was closed to start the experiment. The furnace program setup and muffle furnace temperature setup at $420^{\circ} \mathrm{C}$. Muffle furnace start from room temperature and ramping rate at $20^{\circ} \mathrm{C}$ per minute. Hold at final temperature for 30 minutes. Then the sample was left to cool down at $5^{\circ} \mathrm{C}$ per minute. When temperature went down from 300 to $290^{\circ} \mathrm{C}$ the muffle furnace door was open to transfer the liquid slurry into the reactor chamber for condensation process. During muffle furnace process some light gas escaped out and absorbed by fume hood filter. Muffle furnace experiment run was under in presence of oxygen. $1^{\text {st }}$ step muffle furnace liquid slurry transferred into a steel reactor and set up condensation unit with collection flask unit. This $2^{\text {nd }}$ step process was then heated at temperature ranging from $300-420^{\circ} \mathrm{C}$ to convert the liquid slurry into vapor, and then the vapor travels through a condenser unit with water cooling system and at the end collected liquid fuel or plastic fuel (Shown fig.1, 1). Optimum temperature was for this experiment $370^{\circ} \mathrm{C}$ from start to end of the experiment. Water circulator system was used and cooling water temperature range was $16-17^{\circ} \mathrm{C}$. 
During production period some light hydrocarbon gas was generated which was not condense because of minus boiling point range and gas was methane, ethane, propane and butane. This light gas passed through alkali solution wash for removing contamination from gas. It's called natural gas or light gas. Natural or light gas was storage into Teflon bag and this gas can be reuse for sample heating sources or other purpose. For plastic to produced plastic fuel cleaning purpose a RCI fuel purification system was used to remove water particles and fuel sediments at the end collected final fuel. Produced fuel density is $0.78 \mathrm{gm} / \mathrm{ml}$. By using this process fuel yield percentage is $85 \%$, residue (black coal) yield percentage is $6 \%$ and light gas yield percentage is $6 \%$. During this process no catalyst or any extra chemical because waste plastic pre analysis result showed plastics already contains metal which stimulated the thermal degradation process reaction in which the long chain hydrocarbon break down into short chain hydrocarbon. For that reason this experiment did not use any kind of catalyst. During production period the metal compounds did not come out with liquid fuel as they were leftover residue or black coal inside. Residue or black coal which was hard and color was black has a Btu value.

\section{Results and Discussion}

\subsection{Raw Sample Pre-analysis}

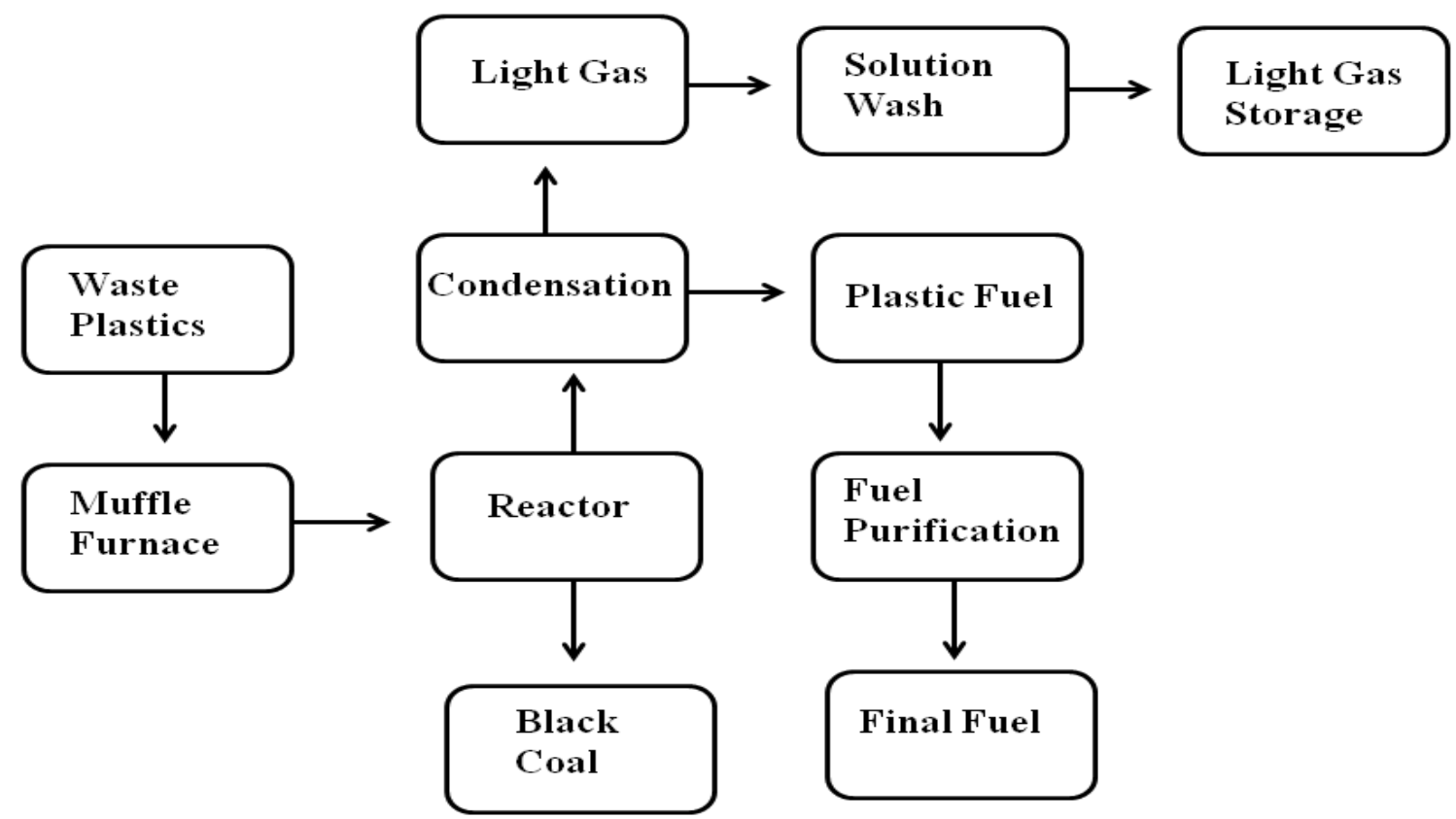

Figure 1. None code waste plastic to fuel production process using muffle furnace through reactor

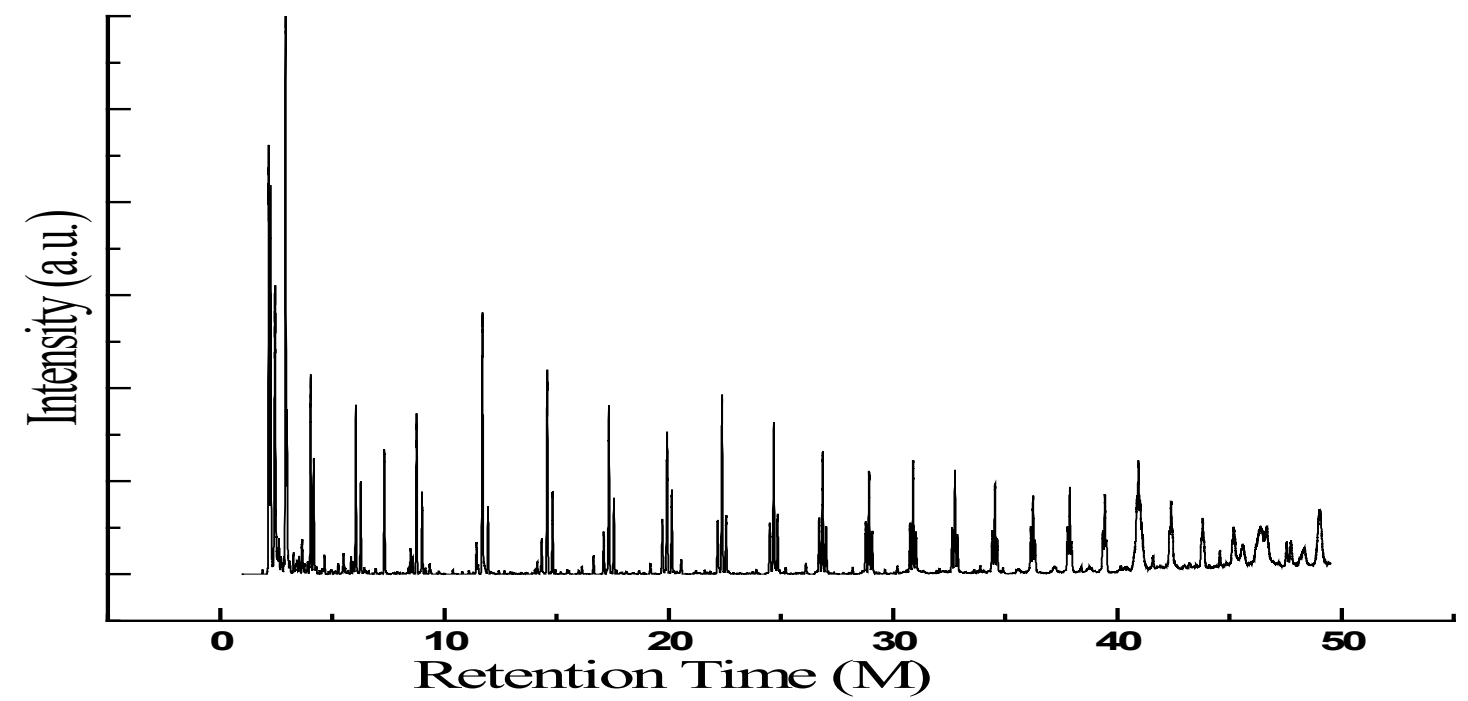

Figure 2. GC/MS chromatogram of none coded waste plastic 
Table 1. GC/MS chromatogram compound list of none coded plastic raw materials

\begin{tabular}{|c|c|c|c|c|c|c|c|}
\hline $\begin{array}{c}\text { Peak } \\
\text { Number }\end{array}$ & $\begin{array}{l}\text { Retention } \\
\text { Time } \\
\text { (M) }\end{array}$ & $\begin{array}{l}\text { Trace } \\
\text { Mass } \\
(\mathbf{m} / \mathbf{z})\end{array}$ & $\begin{array}{l}\text { Compound } \\
\text { Name }\end{array}$ & $\begin{array}{l}\text { Compound } \\
\text { Formula }\end{array}$ & $\begin{array}{l}\text { Molecular } \\
\text { weight }\end{array}$ & Probability \% & $\begin{array}{c}\text { NIST } \\
\text { Library } \\
\text { Number }\end{array}$ \\
\hline 1 & 2.17 & 41 & Cyclopropane & $\mathrm{C}_{3} \mathrm{H}_{6}$ & 42 & 40.3 & 18854 \\
\hline 2 & 2.25 & 41 & 2-Butene, (E)- & $\mathrm{C}_{4} \mathrm{H}_{8}$ & 56 & 19.9 & 105 \\
\hline 3 & 2.46 & 42 & Pentane & $\mathrm{C}_{5} \mathrm{H}_{12}$ & 72 & 43.6 & 114462 \\
\hline 4 & 2.63 & 66 & 1,3-Cyclopentadiene & $\mathrm{C}_{5} \mathrm{H}_{6}$ & 66 & 19.9 & 196 \\
\hline 5 & 2.92 & 56 & $\begin{array}{c}\text { Cyclopropane, 1-ethyl-2-methyl-, } \\
\text { cis- }\end{array}$ & $\mathrm{C}_{6} \mathrm{H}_{12}$ & 84 & 19.8 & 113658 \\
\hline 6 & 2.99 & 41 & Hexane & $\mathrm{C}_{6} \mathrm{H}_{14}$ & 86 & 59.3 & 291337 \\
\hline 7 & 3.28 & 56 & Cyclopentane, methyl- & $\mathrm{C}_{6} \mathrm{H}_{12}$ & 84 & 55.1 & 19308 \\
\hline 8 & 3.45 & 79 & 4-Methylenecyclopentene & $\mathrm{C}_{6} \mathrm{H}_{8}$ & 80 & 14.8 & 210237 \\
\hline 9 & 3.53 & 67 & Cyclopentene, 1-methyl- & $\mathrm{C}_{6} \mathrm{H}_{10}$ & 82 & 13.2 & 107747 \\
\hline 10 & 3.64 & 78 & Benzene & $\mathrm{C}_{6} \mathrm{H}_{6}$ & 78 & 67.8 & 228009 \\
\hline 11 & 4.04 & 56 & 1-Heptene & $\mathrm{C}_{7} \mathrm{H}_{14}$ & 98 & 25.0 & 19704 \\
\hline 12 & 4.18 & 43 & Heptane & $\mathrm{C}_{7} \mathrm{H}_{16}$ & 100 & 63.7 & 61276 \\
\hline 13 & 4.55 & 81 & $\begin{array}{l}\text { Cyclopentane, } \\
\text { 1-methyl-2-methylene- }\end{array}$ & $\mathrm{C}_{7} \mathrm{H}_{12}$ & 96 & 30.4 & 62523 \\
\hline 14 & 4.65 & 55 & Cyclohexane, methyl- & $\mathrm{C}_{7} \mathrm{H}_{14}$ & 98 & 54.4 & 118503 \\
\hline 15 & 5.28 & 69 & 1-Heptene, 4-methyl- & $\mathrm{C}_{8} \mathrm{H}_{16}$ & 112 & 18.0 & 113433 \\
\hline 16 & 5.50 & 91 & 1,3,5-Cycloheptatriene & $\mathrm{C}_{7} \mathrm{H}_{8}$ & 92 & 15.6 & 230230 \\
\hline 17 & 5.58 & 81 & Cyclohexene, 3-methyl- & $\mathrm{C}_{7} \mathrm{H}_{12}$ & 96 & 29.5 & 139433 \\
\hline 18 & 5.84 & 41 & 1,7-Octadiene & $\mathrm{C}_{8} \mathrm{H}_{14}$ & 110 & 32.2 & 27635 \\
\hline 19 & 5.94 & 56 & 1-Heptene, 2-methyl- & $\mathrm{C}_{8} \mathrm{H}_{16}$ & 112 & 59.5 & 231891 \\
\hline 20 & 6.05 & 55 & 1-Octene & $\mathrm{C}_{8} \mathrm{H}_{16}$ & 112 & 15.5 & 191147 \\
\hline 21 & 6.27 & 43 & Heptane, 2,4-dimethyl- & $\mathrm{C}_{9} \mathrm{H}_{20}$ & 128 & 22.5 & 155382 \\
\hline 22 & 7.33 & 43 & 2,4-Dimethyl-1-heptene & $\mathrm{C}_{9} \mathrm{H}_{18}$ & 126 & 31.4 & 113516 \\
\hline 23 & 8.39 & 55 & Cyclohexanemethanol & $\mathrm{C}_{7} \mathrm{H}_{14} \mathrm{O}$ & 114 & 28.1 & 298460 \\
\hline 24 & 8.49 & 54 & 1,8-Nonadiene & $\mathrm{C}_{9} \mathrm{H}_{16}$ & 124 & 43.0 & 107523 \\
\hline 25 & 8.59 & 56 & 1-Decene, 2-methyl- & $\mathrm{C}_{11} \mathrm{H}_{22}$ & 154 & 8.38 & 61173 \\
\hline 26 & 8.76 & 55 & 1-Nonene & $\mathrm{C}_{9} \mathrm{H}_{18}$ & 126 & 12.4 & 107756 \\
\hline 27 & 9.01 & 43 & Nonane & $\mathrm{C}_{9} \mathrm{H}_{20}$ & 128 & 35.4 & 2665 \\
\hline 28 & 9.35 & 83 & $\begin{array}{c}\text { Cyclopentane, } \\
\text { 1-methyl-2-(2-propenyl)-, trans- }\end{array}$ & $\mathrm{C}_{9} \mathrm{H}_{16}$ & 124 & 56.9 & 26931 \\
\hline 29 & 10.38 & 62 & 1-Hexene, 3,3,5-trimethyl- & $\mathrm{C}_{9} \mathrm{H}_{18}$ & 126 & 7.56 & 63168 \\
\hline 30 & 10.53 & 57 & 1-Hexene, 5,5-dimethyl- & $\mathrm{C}_{8} \mathrm{H}_{16}$ & 112 & 12.7 & 60979 \\
\hline 31 & 11.44 & 55 & 1,9-Decadiene & $\mathrm{C}_{10} \mathrm{H}_{18}$ & 138 & 32.6 & 118291 \\
\hline 32 & 11.70 & 55 & 1-Decene & $\mathrm{C}_{10} \mathrm{H}_{20}$ & 140 & 6.11 & 107686 \\
\hline 33 & 11.95 & 43 & Decane & $\mathrm{C}_{10} \mathrm{H}_{22}$ & 142 & 38.2 & 291484 \\
\hline 34 & 14.34 & 55 & 1,10-Undecadiene & $\mathrm{C}_{11} \mathrm{H}_{20}$ & 152 & 20.8 & 227587 \\
\hline 35 & 14.59 & 55 & E-1 1,13-Tetradecadien-1-ol & $\mathrm{C}_{14} \mathrm{H}_{26} \mathrm{O}$ & 210 & 4.98 & 131003 \\
\hline 36 & 14.83 & 43 & Undecane & $\mathrm{C}_{11} \mathrm{H}_{24}$ & 156 & 26.2 & 249213 \\
\hline 37 & 16.65 & 56 & Undecane, 5-methylene- & $\mathrm{C}_{12} \mathrm{H}_{24}$ & 168 & 6.17 & 62764 \\
\hline 38 & 17.11 & 55 & 1,11-Dodecadiene & $\mathrm{C}_{12} \mathrm{H}_{22}$ & 166 & 13.0 & 232664 \\
\hline 39 & 17.33 & 55 & 1-Dodecene & $\mathrm{C}_{12} \mathrm{H}_{24}$ & 168 & 9.06 & 107688 \\
\hline 40 & 17.56 & 57 & Dodecane & $\mathrm{C}_{12} \mathrm{H}_{26}$ & 170 & 29.5 & 291499 \\
\hline 41 & 19.19 & 56 & $\begin{array}{l}\text { Cyclopentane, } \\
\text { 3-hexyl-1,1-dimethyl- }\end{array}$ & $\mathrm{C}_{13} \mathrm{H}_{26}$ & 182 & 5.22 & 62298 \\
\hline 42 & 19.72 & 55 & 1,12-Tridecadiene & $\mathrm{C}_{13} \mathrm{H}_{24}$ & 180 & 6.35 & 158325 \\
\hline 43 & 19.94 & 41 & 1-Tridecene & $\mathrm{C}_{13} \mathrm{H}_{26}$ & 182 & 6.74 & 107768 \\
\hline 44 & 20.14 & 57 & Tridecane & $\mathrm{C}_{13} \mathrm{H}_{28}$ & 184 & 8.01 & 114282 \\
\hline 45 & 22.19 & 55 & 1,11-Dodecadiene & $\mathrm{C}_{12} \mathrm{H}_{22}$ & 166 & 7.37 & 232664 \\
\hline 46 & 22.38 & 55 & Cyclotetradecane & $\mathrm{C}_{14} \mathrm{H}_{28}$ & 196 & 9.86 & 61052 \\
\hline 47 & 22.57 & 57 & Tetradecane & $\mathrm{C}_{14} \mathrm{H}_{30}$ & 198 & 13.9 & 229858 \\
\hline 48 & 24.51 & 41 & 1,13-Tetradecadiene & $\mathrm{C}_{14} \mathrm{H}_{26}$ & 194 & 7.54 & 62224 \\
\hline
\end{tabular}




\begin{tabular}{|c|c|c|c|c|c|c|c|}
\hline 49 & 24.69 & 55 & 1-Hexadecene & $\mathrm{C}_{16} 6^{\mathrm{H}_{32}}$ & 224 & 6.99 & 118882 \\
\hline 50 & 24.86 & 57 & Pentadecane & $\mathrm{C}_{15} \mathrm{H}_{32}$ & 212 & 21.4 & 107761 \\
\hline 51 & 26.12 & 56 & Cyclodecane, methyl- & $\mathrm{C}_{11} \mathrm{H}_{22}$ & 154 & 6.90 & 61039 \\
\hline 52 & 26.71 & 81 & 1,15-Pentadecanediol & $\mathrm{C}_{15} \mathrm{H}_{32} \mathrm{O}_{2}$ & 244 & 8.53 & 113063 \\
\hline 53 & 26.87 & 83 & 1-Hexadecene & $\mathrm{C}_{16} 6^{\mathrm{H}_{32}}$ & 224 & 5.23 & 118882 \\
\hline 54 & 27.02 & 57 & Hexadecane & $\mathrm{C}_{16} 6^{\mathrm{H}_{34}}$ & 226 & 13.2 & 107738 \\
\hline 55 & 28.78 & 55 & 1,15-Pentadecanediol & $\mathrm{C}_{15} \mathrm{H}_{32} \mathrm{O}_{2}$ & 244 & 13.7 & 113063 \\
\hline 56 & 28.93 & 83 & E-14-Hexadecenal & $\mathrm{C}_{16} \mathrm{H}_{30} \mathrm{O}$ & 238 & 7.18 & 130980 \\
\hline 57 & 29.08 & 57 & Heptadecane & $\mathrm{C}_{17} \mathrm{H}_{36}$ & 240 & 13.7 & 107308 \\
\hline 58 & 30.77 & 55 & 1,14-Tetradecanediol & $\mathrm{C}_{14} \mathrm{H}_{30} \mathrm{O}_{2}$ & 230 & 10.8 & 73582 \\
\hline 59 & 30.90 & 83 & 1-Nonadecene & $\mathrm{C}_{19}{ } \mathrm{H}_{38}$ & 266 & 8.36 & 113626 \\
\hline 60 & 31.02 & 71 & Nonadecane & $\mathrm{C}_{19}{ }^{\mathrm{H}_{40}}$ & 268 & 6.75 & 114098 \\
\hline 61 & 32.64 & 55 & 1,14-Tetradecanediol & $\mathrm{C}_{14} \mathrm{H}_{30} \mathrm{O}_{2}$ & 230 & 13.0 & 73582 \\
\hline 62 & 32.77 & 55 & 1-Nonadecene & $\mathrm{C}_{19}{ } \mathrm{H}_{38}$ & 266 & 6.86 & 113626 \\
\hline 63 & 32.88 & 57 & Nonadecane & $\mathrm{C}_{19}{ } \mathrm{H}_{40}$ & 268 & 10.8 & 114098 \\
\hline 64 & 34.34 & 55 & 1,14-Tetradecanediol & $\mathrm{C}_{14} \mathrm{H}_{30} \mathrm{O}_{2}$ & 230 & 13.9 & 73582 \\
\hline 65 & 34.55 & 97 & 10-Heneicosene $(c, t)$ & $\mathrm{C}_{21} \mathrm{H}_{42}$ & 294 & 7.25 & 113073 \\
\hline 66 & 34.66 & 57 & Eicosane & $\mathrm{C}_{20} \mathrm{H}_{42}$ & 282 & 9.94 & 149863 \\
\hline 67 & 36.15 & 55 & 1,15-Pentadecanediol & $\mathrm{C}_{15} \mathrm{H}_{32} \mathrm{O}_{2}$ & 244 & 14.1 & 113063 \\
\hline 68 & 36.25 & 43 & 10-Heneicosene $(c, t)$ & $\mathrm{C}_{21} \mathrm{H}_{42}$ & 294 & 10.9 & 113073 \\
\hline 69 & 36.34 & 57 & Octadecane, 2-methyl- & $\mathrm{C}_{19}{ }^{\mathrm{H}_{40}}$ & 268 & 5.24 & 114071 \\
\hline 70 & 37.79 & 55 & 1,15-Pentadecanediol & $\mathrm{C}_{15} \mathrm{H}_{32} \mathrm{O}_{2}$ & 244 & 9.93 & 113063 \\
\hline 71 & 37.88 & 97 & 10-Heneicosene $(c, t)$ & $\mathrm{C}_{21} \mathrm{H}_{42}$ & 294 & 8.89 & 113073 \\
\hline 72 & 39.45 & 55 & 1-Docosene & $\mathrm{C}_{22} \mathrm{H}_{44}$ & 308 & 8.75 & 113878 \\
\hline 73 & 40.95 & 69 & 1-Eicosanol & $\mathrm{C}_{20} \mathrm{H}_{42} \mathrm{O}$ & 298 & 11.4 & 113075 \\
\hline 74 & 42.41 & 57 & 1-Eicosanol & $\mathrm{C}_{20} \mathrm{H}_{42} \mathrm{O}$ & 298 & 12.3 & 113075 \\
\hline 75 & 43.41 & 83 & 1-Eicosanol & $\mathrm{C}_{20} \mathrm{H}_{42} \mathrm{O}$ & 298 & 7.79 & 113075 \\
\hline 76 & 46.67 & 83 & 1-Eicosanol & $\mathrm{C}_{20} \mathrm{H}_{42} \mathrm{O}$ & 298 & 6.02 & 113075 \\
\hline 77 & 74.55 & 69 & $\begin{array}{c}\text { 11-Dodecen-1-ol, } \\
\text { 2,4,6-trimethyl-, (R,R,R)- }\end{array}$ & $\mathrm{C}_{15} \mathrm{H}_{30} \mathrm{O}$ & 226 & 7.64 & 10749 \\
\hline 78 & 47.74 & 69 & Oxirane, tetradecyl- & $\mathrm{C}_{16} \mathrm{H}_{32} \mathrm{O}$ & 240 & 7.83 & 75831 \\
\hline 79 & 49.01 & 69 & $\begin{array}{c}\text { 11-Dodecen-1-ol, } \\
\text { 2,4,6-trimethyl-, (R,R,R)- }\end{array}$ & $\mathrm{C}_{15} \mathrm{H}_{30} \mathrm{O}$ & 226 & 8.10 & 31254 \\
\hline
\end{tabular}

Perkin Elmer GC-MS pyroprobe analysis of non-coded raw solid waste plastics inside of pyroprobe raw solid waste plastics (fig.2, $r$ and table 1, I) turns into volatile gas with high temperature at $1200{ }^{\circ} \mathrm{C}$ and that volatile gas passed through the column to gas chromatography, helium $(\mathrm{He})$ is used as a carrier gas and then sends the volatile gas to the mass spectroscopy and in mass compounds are detected according to the boiling point of individual compound and among those only several compounds are introduced as well elaborated in the analysis. In accordance with the retention time and trace masses numerous different types of hydrocarbon compound and benzene derivatives compounds are appeared in the analys is result index. Many compounds are emerged on the analys is carbon range $C_{3}$ to $C_{22}$. In the initial state of the analys is index according to the retention time such as retention time 2.17 and trace mass 41 ,compound is single bond Cyclopropane $\left(\mathrm{C}_{3} \mathrm{H}_{6}\right)$, retention time 2.25 and trace mass 41 compound is double bond
Butene, $(\mathrm{E})-\left(\mathrm{C}_{4} \mathrm{H}_{8}\right)$, retention time 2.46 and trace mass 42 , compound is single bond Pentane, $\left(\mathrm{C}_{5} \mathrm{H}_{12}\right)$, retention time 2.63 and trace mass 66, compound is 1-3 Cyclopentadiene $\left(\mathrm{C}_{5} \mathrm{H}_{6}\right)$, retention time 2.92 and trace mass 56 , compound is Cyclopropane, 1-ethyl-2-methyl-,c is- $\left(\mathrm{C}_{6} \mathrm{H}_{12}\right)$, retention time 2.99 and trace mass 41 , compound is single bond Hexane $\left(\mathrm{C}_{6} \mathrm{H}_{14}\right)$, retention time 3.28 and trace mass 56 , compound is Cyclopentane, methyl- $\left(\mathrm{C}_{6} \mathrm{H}_{12}\right)$, retention time 3.45 and trace mass 79 , co mpound name is 4-Methylencyclopentene $\left(\mathrm{C}_{6} \mathrm{H}_{8}\right)$, retention time 3.64 and trace mass 78 , compound is Benzene $\left(\mathrm{C}_{6} \mathrm{H}_{6}\right)$, retention time 4.55 and trace mass 81 , compound is Cyclopentane,1-methyl-2-methylene $\left(\mathrm{C}_{7} \mathrm{H}_{12}\right)$, retention time 4.65 and trace mass 55,compound is Benzene group Cyclohexane, methyl- $\left(\mathrm{C}_{7} \mathrm{H}_{14}\right)$, retention time 5.94 and trace mass 56, compound is 1-Heptene,-2-methyl- $\left(\mathrm{C}_{8} \mathrm{H}_{16}\right)$, retention time 6.05 and trace mass 55, compound is double bond 1-Octene $\left(\mathrm{C}_{8} \mathrm{H}_{16}\right)$, retention time 7.33 and trace mass 43, compound is double bonding 2,4-Dimethyl-1-heptene 
$\left(\mathrm{C}_{9} \mathrm{H}_{18}\right)$, retention time 8.76 and trace mass 55 ,compound is double bond 1-Nonene $\left(\mathrm{C}_{9} \mathrm{H}_{18}\right)$, retention time 10.38 and trace mass 62, compound is double bond 1-Hexene,3,3,5trimethyl- $\left(\mathrm{C}_{9} \mathrm{H}_{18}\right)$, retention time 11.95 and trace mass 43 , compound is Decane $\left(\mathrm{C}_{10} \mathrm{H}_{22}\right)$, retention time 14.83 and trace mass 43, compound name is single bond Undecane $\left(\mathrm{C}_{11} \mathrm{H}_{24}\right)$, retention time 17.56 and trace mass 57 , co mpound is single bond Dodecane $\left(\mathrm{C}_{12} \mathrm{H}_{26}\right)$, retention time 19.94 and trace mass 41 , compound is double bond Tridecene $\left(\mathrm{C}_{13} \mathrm{H}_{26}\right)$, retention time 22.57 and trace mass 57 , compound is single bond Tetradecane $\left(\mathrm{C}_{14} \mathrm{H}_{30}\right)$, retention time 24.86 and trace mass 57, compound is single bond Pentadecane $\left(\mathrm{C}_{15} \mathrm{H}_{32}\right)$, As well retention time 30.77 and trace mass 55 , compound is 1,14-Tetradecanediol $\left(\mathrm{C}_{14} \mathrm{H}_{30} \mathrm{O}_{2}\right)$, here appearing that oxygen compound are produced because in the reactor during reaction phase oxygen induce from steam and water, retention time 31.02 and trace mass 71 , compound is single bond Nonadecane $\left(\mathrm{C}_{19} \mathrm{H}_{40}\right)$, retention time 32.77 and trace mass 55, compound is double bond Nonadecene $\left(\mathrm{C}_{19} \mathrm{H}_{32}\right)$, retention time 34.34 and trace mass 55, compound is oxygenated 1,14-Tetradecanediol $\left(\mathrm{C}_{14} \mathrm{H}_{30} \mathrm{O}_{2}\right)$, retention time 36.15 and trace mass 55, compound is oxygenated 1,15Pentadecanediol $\left(\mathrm{C}_{15} \mathrm{H}_{32} \mathrm{O}_{2}\right)$. At the last phase of the analys is index retention time 37.79 and trace mass 55, compound is 1 , 14-Tetradecanediol $\left(\mathrm{C}_{14} \mathrm{H}_{30} \mathrm{O}_{2}\right)$, retention time 39.45 and trace mass 55, compound is double bond 1-Dococsene $\left(\mathrm{C}_{22} \mathrm{H}_{44}\right)$, retention time 40.95 and trace mass 69 , compound is 1-Eicosanol $\left(\mathrm{C}_{20} \mathrm{H}_{42} \mathrm{O}\right)$, retention time 46.67 and trace mass 83 , compound is 1-Eicosanol $\left(\mathrm{C}_{20} \mathrm{H}_{42} \mathrm{O}\right)$ and ultimately retention time 47.74 and trace mass 69 compound is Oxirane, tetradecyl $\left(\mathrm{C}_{16} \mathrm{H}_{32} \mathrm{O}\right)$ etc.

Non-Code waste plastic was analyzed by FT-IR ATR (Attenuated Total Reflectance) KRS -5 diamond plate. In according to the wave number following types of functional groups are appeared in the analysis. According to the wave number $2916.05 \mathrm{c} \mathrm{m}^{-1}$, functional group is $\mathrm{CH}_{2}$, wave number $2848.53 \mathrm{~cm}^{-1}$, functional group is $\mathrm{C}-\mathrm{CH}_{3}$, wave number $1463.95 \mathrm{c} \mathrm{m}^{-1}$, functional group is $\mathrm{CH}_{3}$ as we as wave number $1376.92 \mathrm{~cm}^{-1}$, functional group is also $\mathrm{CH}_{3}$ and ultimately wave number $718.84 \mathrm{~cm}^{-1}$, functional group is $-\mathrm{CH}=\mathrm{CH}$ - (cis) respectively. For Each and every wave number band energy was calculated accordingly. Energy, E=hcW, where $\mathrm{h}=$ Plank's Constant, $\mathrm{c}=$ speed of light and $\mathrm{W}=$ wave number, such as wave number $2916.05 \mathrm{~cm}^{-1}\left(\mathrm{CH}_{2}\right)$, energy, $\mathrm{E}=5.79 \times 10^{-20} \mathrm{~J}$, then wave number $2848.53 \mathrm{~cm}^{-1}\left(\mathrm{C}-\mathrm{CH}_{3}\right)$ energy, $\mathrm{E}=5.65 \times 10^{-20} \mathrm{~J}$, wave number $\left.1463.95 \mathrm{~cm}^{-1} \mathrm{CH}_{3}\right)$ energy, $\mathrm{E}=2.90 \times 10^{-20} \mathrm{~J}$, wave number $1376.92 \mathrm{~cm}^{-1}\left(\mathrm{CH}_{3}\right)$ energy, $\mathrm{E}=2.75 \times 10^{-20} \mathrm{~J}$ and eventually wave number 718.84 $\mathrm{cm}^{-1}(-\mathrm{CH}=\mathrm{CH}-(\mathrm{c}$ is $))$ energy, $\mathrm{E}=1.42 \times 10^{-20} \mathrm{~J}$.

EA-2400 analysis result showed from non-coded waste plastic materials carbon present $85.3 \%$, Hydrogen present $13.4 \%$ and Nitrogen present $0.35 \%$ and EA-2400 was analysis sample CHN mode and carrier has was Oxygen, Helium and Nitrogen. TGA (Pyris-1) was use for raw material onset temperature and inflection point temperature determination and analysis temperature setup was for TGA $50-800^{\circ} \mathrm{C}$ and temperature increase rate was $15^{\circ} \mathrm{C} /$ minute and sample was use for analys is $2.9 \mathrm{mg}$. Carrier gas was use heliu m at $20 \mathrm{ml} / \mathrm{min}$. Non-coded waste plastics was analys is by TGA and graph obtain results showed inflection point temperature $413.89^{\circ} \mathrm{C}$ and onset temperature was $387.81^{\circ} \mathrm{C}$. From those temperatures was determined liquefaction temperature set up for waste plastic to liquid fuel production process. This analysis temperature was helping us to raw materials fully volatile percentage and leftover residue present after thermal degradation process.

\subsection{Liquid Fuel Analysis}

From FT-IR analysis (Spectrum 100 Perkin Elmer) we found functional group (see fig.3, $r$ and table $2, r$ ) and from functional group we found some band energy. FT-IR (Fourier Transform Infra-red Spectroscopy) analys is of Non-Code plastic to fuel is noticed following types of functional group appeared such as at wave number 3611.17 $\mathrm{cm}^{-1}$, compound is Free $\mathrm{OH}$, wave number $3426.25 \mathrm{~cm}^{-1}$, compound is intermo lecular $\mathrm{H}$ bonds, wave number 3077.64 $\mathrm{cm}^{-1}$,compound is $\mathrm{H}$ bonded $\mathrm{NH}$, wave number 2857.17 $\mathrm{cm}^{-1}, 2730.97 \mathrm{~cm}^{-1}, 2671.63 \mathrm{~cm}^{-1}$ compound is $\mathrm{C}-\mathrm{CH}_{3}$, again appearing descending way wave number 1821.49 $\mathrm{cm}^{-1}, 1718.97 \mathrm{~cm}^{-1}$ compound is Non-Conjugated, wave number $1641.65 \mathrm{~cm}^{-1}$,compound is conjugated, wave number $1440.10 \mathrm{~cm}^{-1}$ and $1377.72 \mathrm{~cm}^{-1}$ compound is $\mathrm{CH}_{3}$, wave number $992.10 \mathrm{~cm}^{-1}$ compound is $-\mathrm{CH}=\mathrm{CH}_{2}$, wave number $965.32 \mathrm{~cm}^{-1}$, compound is $-\mathrm{CH}=\mathrm{CH}$-(trans), wave number 908.03 compound is $-\mathrm{CH}=\mathrm{CH}_{2}$, wave number 887.97 $\mathrm{cm}^{-1}$, compound is $\mathrm{C}=\mathrm{CH}_{2}$ and ultimately wave number $721.79 \mathrm{~cm}^{-1}$,compound is $-\mathrm{CH}=\mathrm{CH}-(\mathrm{c}$ is $)$ respectively. Energy value are calculated using energy formula that is $\mathrm{E}=\mathrm{hcw}$, where $\mathrm{E}=$ energy, $\mathrm{h}=$ plank constant, $\omega=$ frequency number/wave number. Functional group $\mathrm{C}-\mathrm{CH}_{3}$,calculated energy value is $5.67 \times 10^{-20} \mathrm{~J}$, functional group $\mathrm{CH}_{3}$,energy value is $2.86 \times 10^{-20} \mathrm{~J}$ and ultimately functional group $\mathrm{C}=\mathrm{CH}_{2}$, calculated energy value is $1.76 \times 10^{-20} \mathrm{~J}$. Euclidean Search Hit List: 0.384 F37460 2,5-DIHYDROXYACETOPHENONE, 0.299 F06640 4-AMINO-ACETOPHENONE, $\quad 0.289$ F00508 ETHYL ACETOHYDROXAMATE, 0.284 F91080 TRICHLOROA CETONITRILE, 0.266 F65155 2-METHO XYPHENYLACETONITRILE, 0.249 F22850 4-CHLORO ACETOPHENONE, 0.239 F54150 2-HYDROXYACETOP HENONE, 0.239 F65470 3-METHYLA CETOPHENONE, 0.224 F35038 1,1-DICHLOROACETONE, 0.219 F80072 BIS (3,5,5-TRIMETHYL HEXYL) PHTHALATE ( Perkin Elmer FT-IR tutorial library) 


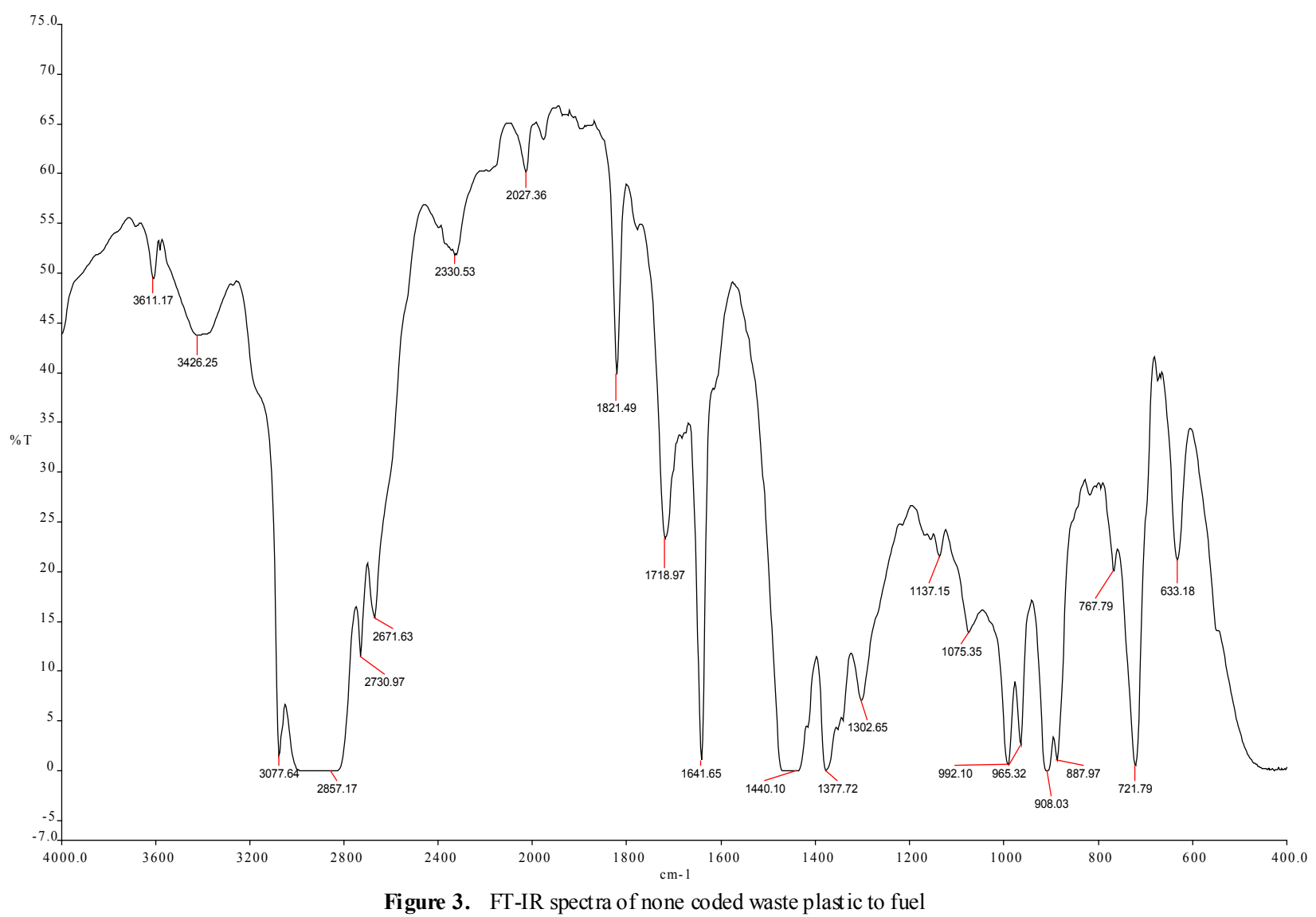

Table 2. FT-IR spectra of none coded waste plast ic to fuel functional group

\begin{tabular}{|c|c|c|c|c|c|}
\hline Number of Wave & $\begin{array}{l}\text { Wave Number } \\
\left(\mathrm{cm}^{-1}\right)\end{array}$ & $\begin{array}{c}\text { Functional Group } \\
\text { Name }\end{array}$ & Number of Wave & $\begin{array}{c}\text { Wave Number } \\
\left(\mathrm{cm}^{-1}\right)\end{array}$ & $\begin{array}{c}\text { Fractional Group } \\
\text { Name }\end{array}$ \\
\hline 1 & 3611.17 & Free $\mathrm{OH}$ & 11 & 1641.65 & Conjugated \\
\hline 2 & 3426.25 & $\begin{array}{l}\text { Inter Molecular } \mathrm{H} \\
\text { bonds }\end{array}$ & 12 & 1440.10 & $\mathrm{CH}_{3}$ \\
\hline 3 & 3077.64 & $\mathrm{H}$ bonded $\mathrm{NH}$ & 13 & 1377.72 & $\mathrm{CH}_{3}$ \\
\hline 4 & 2857.17 & $\mathrm{C}-\mathrm{CH}_{3}$ & 17 & 992.10 & $-\mathrm{CH}=\mathrm{CH}_{2}$ \\
\hline 5 & 2730.97 & $\mathrm{C}-\mathrm{CH}_{3}$ & 18 & 965.32 & $-\mathrm{CH}=\mathrm{CH}-($ trans $)$ \\
\hline 6 & 2671.63 & $\mathrm{C}-\mathrm{CH}_{3}$ & 19 & 908.03 & $-\mathrm{CH}=\mathrm{CH}_{2}$ \\
\hline 9 & 1821.49 & Non-Conjugated & 20 & 887.97 & $\mathrm{C}=\mathrm{CH}_{2}$ \\
\hline 10 & 1718.97 & Non-Conjugated & 22 & 721.79 & $-\mathrm{CH}=\mathrm{CH}-(\mathrm{cis})$ \\
\hline
\end{tabular}

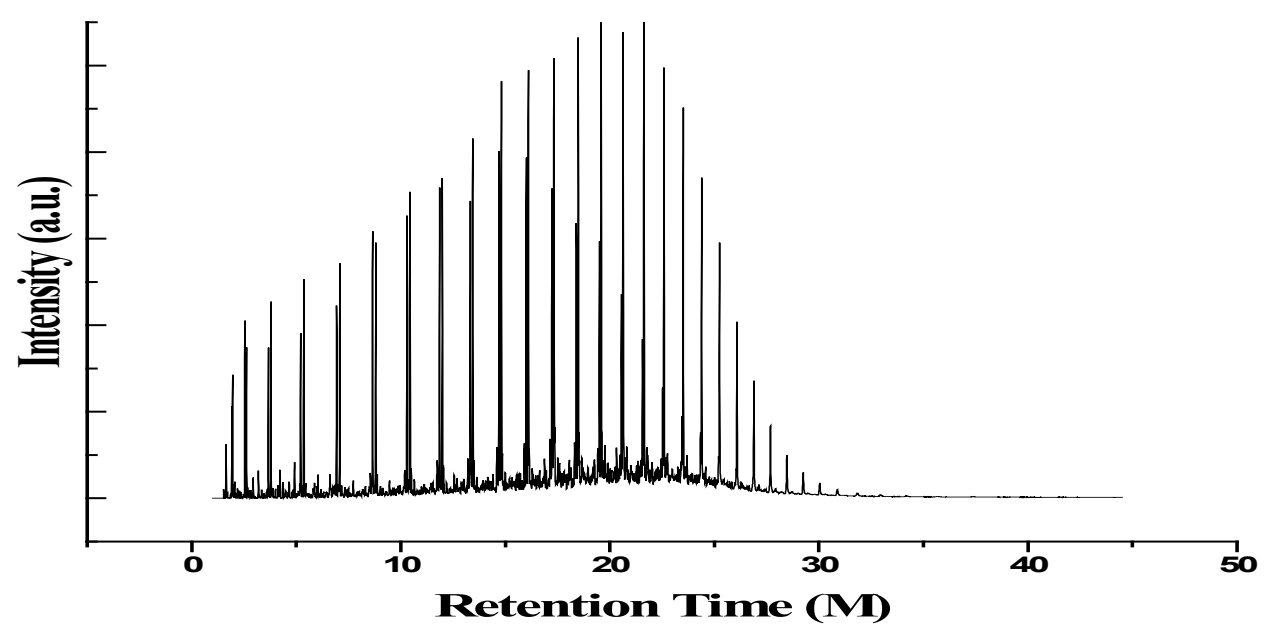

Figure 4. GC/MS Chromatogram of non-coded waste plastic to fuel 
Table 3. Non-coded waste plastic to fuel GC/MS chromat ogram compound list

\begin{tabular}{|c|c|c|c|c|c|c|c|}
\hline $\begin{array}{c}\text { Peak } \\
\text { Number }\end{array}$ & $\begin{array}{l}\text { Retention } \\
\text { Time } \\
\text { (M) }\end{array}$ & $\begin{array}{l}\text { Trace } \\
\text { Mass } \\
(\mathbf{m} / \mathbf{z})\end{array}$ & $\begin{array}{l}\text { Compound } \\
\text { Name }\end{array}$ & $\begin{array}{l}\text { Compound } \\
\text { Formula }\end{array}$ & $\begin{array}{l}\text { Mole cular } \\
\text { Weight }\end{array}$ & $\begin{array}{c}\text { CAS } \\
\text { Number }\end{array}$ & $\begin{array}{c}\text { Carbon-Carbon } \\
\text { Bond }\end{array}$ \\
\hline 1 & 1.53 & 39 & Propane & $\mathrm{C}_{3} \mathrm{H}_{8}$ & 44 & $74-98-6$ & Single \\
\hline 2 & 1.65 & 43 & Butane & $\mathrm{C}_{4} \mathrm{H}_{10}$ & 58 & $106-97-8$ & Single \\
\hline 3 & 1.91 & 42 & Cyclopropane, ethyl- & $\mathrm{C}_{5} \mathrm{H}_{10}$ & 70 & $1191-96-4$ & Single \\
\hline 4 & 1.95 & 43 & Pentane & $\mathrm{C}_{5} \mathrm{H}_{12}$ & 72 & $109-66-0$ & Single \\
\hline 5 & 2.54 & 41 & 1-Hexene & $\mathrm{C}_{6} \mathrm{H}_{12}$ & 84 & $592-41-6$ & Double \\
\hline 6 & 2.62 & 41 & Hexane & $\mathrm{C}_{6} \mathrm{H}_{14}$ & 86 & $110-54-3$ & Single \\
\hline 7 & 3.19 & 67 & Cyclopentene, 1-methyl- & $\mathrm{C}_{6} \mathrm{H}_{10}$ & 82 & $693-89-0$ & Double \\
\hline 8 & 3.66 & 41 & 1-Heptene & $\mathrm{C}_{7} \mathrm{H}_{14}$ & 98 & $592-76-7$ & Double \\
\hline 9 & 3.79 & 43 & Heptane & $\mathrm{C}_{7} \mathrm{H}_{16}$ & 100 & $142-82-5$ & Single \\
\hline 10 & 4.22 & 83 & Cyclohexane, methyl- & $\mathrm{C}_{7} \mathrm{H}_{14}$ & 98 & $108-87-2$ & $\begin{array}{l}\text { Benzene group / } \\
\text { Single }\end{array}$ \\
\hline 11 & 4.92 & 81 & Cyclohexene, 1-methyl- & $\mathrm{C}_{7} \mathrm{H}_{12}$ & 96 & $591-49-1$ & $\begin{array}{l}\text { Benzene group / } \\
\text { Double }\end{array}$ \\
\hline 12 & 5.21 & 41 & 1-Octene & $\mathrm{C}_{8} \mathrm{H}_{16}$ & 112 & $111-66-0$ & Double \\
\hline 13 & 5.36 & 43 & Octane & $\mathrm{C}_{8} \mathrm{H}_{18}$ & 114 & $111-65-9$ & Single \\
\hline 14 & 6.04 & 83 & Cyclohexane, ethyl- & $\mathrm{C}_{8} \mathrm{H}_{16}$ & 112 & $1678-91-7$ & $\begin{array}{l}\text { Benzene group / } \\
\text { Single }\end{array}$ \\
\hline 15 & 6.61 & 81 & $\begin{array}{l}\text { Cyclohexene, } \\
\text { 1,2-dimethyl- }\end{array}$ & $\mathrm{C}_{8} \mathrm{H}_{14}$ & 110 & $1674-10-8$ & $\begin{array}{l}\text { Benzene group / } \\
\text { Double }\end{array}$ \\
\hline 16 & 6.93 & 41 & 1-Nonene & $\mathrm{C}_{9} \mathrm{H}_{18}$ & 126 & $124-11-8$ & Double \\
\hline 17 & 7.08 & 43 & Nonane & $\mathrm{C}_{9} \mathrm{H}_{20}$ & 128 & $111-84-2$ & Single \\
\hline 18 & 8.55 & 70 & Nonane, 3-methylene- & $\mathrm{C}_{10} \mathrm{H}_{20}$ & 140 & $51655-64-2$ & Double \\
\hline 19 & 8.65 & 41 & 1-Decene & $\mathrm{C}_{10} \mathrm{H}_{20}$ & 140 & $872-05-9$ & Double \\
\hline 20 & 8.80 & 57 & Decane & $\mathrm{C}_{10} \mathrm{H}_{22}$ & 142 & $124-18-5$ & Single \\
\hline 21 & 9.45 & 55 & Cyclodecane & $\mathrm{C}_{10} \mathrm{H}_{20}$ & 140 & $293-96-9$ & Cyclic / Single \\
\hline 22 & 10.19 & 70 & 4-Undecene, (Z)- & $\mathrm{C}_{11} \mathrm{H}_{22}$ & 154 & $821-98-7$ & Double \\
\hline 23 & 10.30 & 41 & 1-Undecene & $\mathrm{C}_{11} \mathrm{H}_{22}$ & 154 & $821-95-4$ & Double \\
\hline 24 & 10.44 & 57 & Undecane & $\mathrm{C}_{11} \mathrm{H}_{24}$ & 156 & $1120-21-4$ & Single \\
\hline 25 & 11.74 & 70 & $\begin{array}{c}\text { 5-Undecene, 9-methyl-, } \\
\text { (Z)- }\end{array}$ & $\mathrm{C}_{12} \mathrm{H}_{24}$ & 168 & $74630-65-2$ & Double \\
\hline 26 & 11.86 & 41 & 1-Dodecene & $\mathrm{C}_{12} \mathrm{H}_{24}$ & 168 & $112-41-4$ & Double \\
\hline 27 & 11.98 & 57 & Dodecane & $\mathrm{C}_{12} \mathrm{H}_{26}$ & 170 & $112-40-3$ & Single \\
\hline 28 & 13.21 & 70 & 5-Tridecene, (E)- & $\mathrm{C}_{13} \mathrm{H}_{26}$ & 182 & $23051-84-5$ & Double \\
\hline 29 & 13.32 & 41 & 1-Tridecene & $\mathrm{C}_{13} \mathrm{H}_{26}$ & 182 & $2437-56-1$ & Double \\
\hline 30 & 13.44 & 57 & Tridecane & $\mathrm{C}_{13} \mathrm{H}_{28}$ & 184 & $629-50-5$ & Single \\
\hline 31 & 14.40 & 57 & Tridecane, 3-methyl- & $\mathrm{C}_{14} \mathrm{H}_{30}$ & 198 & $6418-41-3$ & Single \\
\hline 32 & 14.70 & 41 & 1-Tetradecene & $\mathrm{C}_{14} \mathrm{H}_{28}$ & 196 & $1120-36-1$ & Double \\
\hline 33 & 14.82 & 57 & Tetradecane & $\mathrm{C}_{14} \mathrm{H}_{30}$ & 198 & $629-59-4$ & Single \\
\hline 34 & 15.00 & 55 & 7-Tetradecene & $\mathrm{C}_{14} \mathrm{H}_{28}$ & 196 & $10374-74-0$ & Double \\
\hline 35 & 15.90 & 41 & Z-10-Pentadecen-1-ol & $\mathrm{C}_{15} \mathrm{H}_{30} \mathrm{O}$ & 226 & $\mathrm{~N} / \mathrm{A}$ & OH group/Double \\
\hline 36 & 16.00 & 55 & 1-Pentadecene & $\mathrm{C}_{15} \mathrm{H}_{30}$ & 210 & $13360-61-7$ & Double \\
\hline 37 & 16.10 & 57 & Pentadecane & $\mathrm{C}_{15} \mathrm{H}_{32}$ & 212 & $629-62-9$ & Single \\
\hline 38 & 17.14 & 70 & Cyclohexadecane & $\mathrm{C}_{16} 6^{\mathrm{H}_{32}}$ & 224 & $295-65-8$ & $\begin{array}{l}\text { Cyclic group/ } \\
\text { single }\end{array}$ \\
\hline 39 & 17.23 & 55 & 1-Hexadecene & $\mathrm{C}_{16} \mathrm{H}_{32}$ & 224 & $629-73-2$ & Double \\
\hline 40 & 17.33 & 43 & Hexadecane & $\mathrm{C}_{16}{ }^{\mathrm{H}_{34}}$ & 226 & $544-76-3$ & Single \\
\hline 41 & 18.40 & 55 & E-14-Hexadecenal & $\mathrm{C}_{16} \mathrm{H}_{30} \mathrm{O}$ & 238 & $330207-53-9$ & $\begin{array}{c}\text { Oxygenate / } \\
\text { Double }\end{array}$ \\
\hline 42 & 18.49 & 41 & Heptadecane & $\mathrm{C}_{17} \mathrm{H}_{36}$ & 240 & $629-78-7$ & Single \\
\hline 43 & 19.50 & 55 & E-15-Heptadecenal & $\mathrm{C}_{17} \mathrm{H}_{32} \mathrm{O}$ & 252 & N/A & $\begin{array}{c}\text { Oxygenate / } \\
\text { Double }\end{array}$ \\
\hline
\end{tabular}




\begin{tabular}{cccccccc}
44 & 19.59 & 57 & Octadecane & $\mathrm{C}_{18} \mathrm{H}_{38}$ & 254 & $593-45-3$ & Single \\
45 & 20.56 & 55 & 1-Nonadecene & $\mathrm{C}_{19} \mathrm{H}_{38}$ & 266 & $18435-45-5$ & Double \\
46 & 20.63 & 43 & Eicosane & $\mathrm{C}_{20} \mathrm{H}_{42}$ & 282 & $112-95-8$ & Single \\
47 & 21.56 & 55 & 1-Docosene & $\mathrm{C}_{22} \mathrm{H}_{44}$ & 308 & $1599-67-3$ & Double \\
48 & 22.59 & 57 & Heneicosane & $\mathrm{C}_{21} \mathrm{H}_{44}$ & 296 & $629-94-7$ & Single \\
49 & 25.25 & 57 & Tetracosane & $\mathrm{C}_{24} \mathrm{H}_{50}$ & 338 & $646-31-1$ & Single \\
50 & 26.07 & 57 & Octacosane & $\mathrm{C}_{28} \mathrm{H}_{58}$ & 394 & $630-02-4$ & Single \\
51 & 27.68 & 57 & Heptacosane & $\mathrm{C}_{27} \mathrm{H}_{56}$ & 380 & $593-49-7$ & Single \\
\hline
\end{tabular}

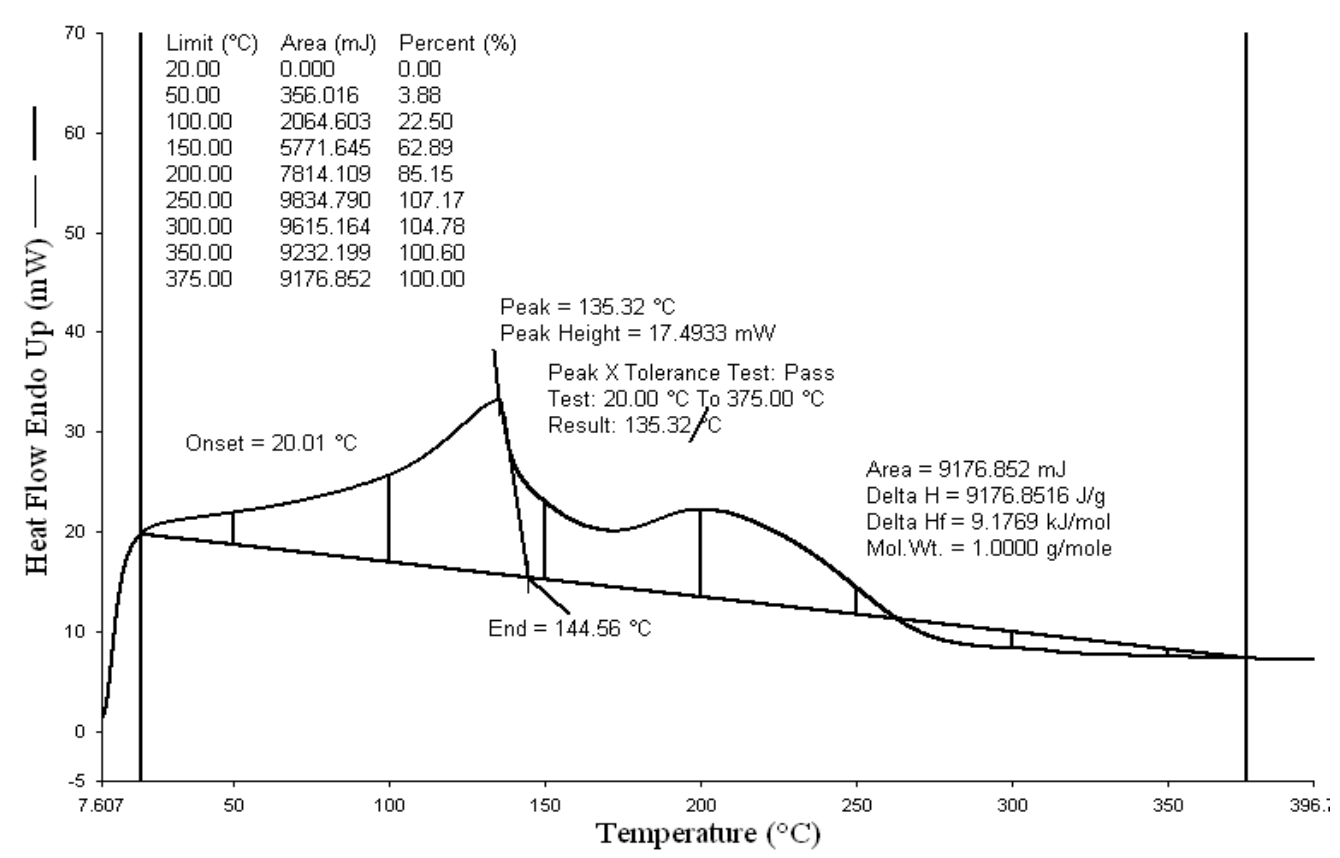

Figure 5. DSC Graph of non code waste plastic to fuel

Perkin Elmer Gas Chromatography and Mass Spectrometer (GC/MS) analysis result of the produced fuel are shown in fig.4, ₹ and table3, r. For GC/MS analysis purpose GC/MS (Perkin Elmer) Clarus 500 series with auto sampler process was used. Carrier gas helium $(\mathrm{He})$ was used in GC/MS capillary column. GC fuel injector port temperature is $300^{\circ} \mathrm{C}$ for making volatile liquid fuel sample to pass though GC column. GC program was setup initial temperature is $40^{\circ} \mathrm{C}$ because all moisture such as water, carbon dioxide helium and nitrogen mass are not above 44 and MS mass detection level we setup 35 to 528. Initial temperature set up to hold for 1 minute for volatile into injector port at $300^{\circ} \mathrm{C}$. Setup final temperature at $350^{\circ} \mathrm{C}$ and temperature ramping rate was $10^{\circ} \mathrm{C}$ per minute and final temperature hold for 18 minutes. Total sample run time was 50 minutes. Capillary column (Perkin Elmer) specification elite-5MS 30 meter length, mmID 0.25, um df 0.5 and temperature capable up to $350{ }^{\circ} \mathrm{C}$. GC-MS Analysis of non-coded waste plastics to fuel in accordance with the retention time and trace masses numerous different types of hydrocarbon compound and benzene derivatives compounds appeared in the analys is result index. Many compounds are emerged on the analys is carbon range $C_{3}$ to $C_{28}$. In the initial state of the analysis index according to the retention time such as retention time 1.53 and trace mass 39 , single bond compound is single bond Propane $\left(\mathrm{C}_{3} \mathrm{H}_{8}\right)$, retention time 1.65 and trace mass 43 compound is single bond Butane $\left(\mathrm{C}_{4} \mathrm{H}_{10}\right)$, retention time 1.91 and trace mass 42 , compound is single bond Cyclopropane, ethyl- $\left(\mathrm{C}_{5} \mathrm{H}_{10}\right)$, retention time 1.95 and trace mass 43 , compound is Pentane $\left(\mathrm{C}_{5} \mathrm{H}_{12}\right)$, retention time 2.54 and trace mass 41 , compound is double bonding 1-Hexene $\left(\mathrm{C}_{6} \mathrm{H}_{12}\right)$, retention time 2.62 and trace mass 41 , compound is single bond Hexane, retention time 3.19 and trace mass 67, compound is double bond Cyclopentene, 1-methyl- $\left(\mathrm{C}_{6} \mathrm{H}_{10}\right)$, retention time 3.66 and trace mass 41,compound name is double bond 1-Heptene $\left(\mathrm{C}_{7} \mathrm{H}_{14}\right)$,retention time 3.79 and trace mass 43 , functional group is single bond Heptane $\left(\mathrm{C}_{7} \mathrm{H}_{16}\right)$, retention time 4.22 and trace mass 83, functional group is Cyclohexane, methyl$\left(\mathrm{C}_{7} \mathrm{H}_{14}\right)$, retention time 4.92 and trace mass 81 ,compound is Benzene group Cyclohexene, 1-methyl- $\left(\mathrm{C}_{7} \mathrm{H}_{12}\right)$, retention time 5.21 and trace mass 41 , compound is 1-Octene $\left(\mathrm{C}_{8} \mathrm{H}_{16}\right)$, retention time 5.36 and trace mass 43 , compound is single bond Octane $\left(\mathrm{C}_{8} \mathrm{H}_{18}\right)$, retention time 6.93 and trace mass 41 ,compound is double bonding 1-Nonene ( $\left.\mathrm{C}_{9} \mathrm{H}_{18}\right)$, retention time 7.08 and trace mass 43 ,compound is single bond Nonane $\left(\mathrm{C}_{9} \mathrm{H}_{20}\right)$, retention time 8.80 and trace mass 57 , compound is single bond Decane $\left(\mathrm{C}_{10} \mathrm{H}_{22}\right)$, retention time 9.45 and trace mass 55, compound is cyclic/single bond Cyclodecane $\left(\mathrm{C}_{10} \mathrm{H}_{20}\right)$, retention time 10.44 and trace mass 
57, compound name is single bond Undecene $\left(\mathrm{C}_{11} \mathrm{H}_{22}\right)$, retention time 11.98 and trace mass 57, compound is single bond Dodecane $\left(\mathrm{C}_{12} \mathrm{H}_{26}\right)$, retention time 13.44 and trace mass 57, compound is single bond Tridecane $\left(\mathrm{C}_{13} \mathrm{H}_{28}\right)$, retention time 14.82 and trace mass compound is single bond Tetradecane $\left(\mathrm{C}_{14} \mathrm{H}_{30}\right)$, retention time 15.90 and trace mass 41 , compound is $\mathrm{OH}$ group/double bond Z-10-Pentadecen-1-ol $\left(\mathrm{C}_{14} \mathrm{H}_{30} \mathrm{O}\right)$, here appearing that oxygen compound are produced because in the reactor during reaction phase oxygen induce from steam and water. As well retention time 16.00 and trace mass 55, compound is double bonding 1-Pentadecene $\left(\mathrm{C}_{15} \mathrm{H}_{32}\right)$, retention time 17.33 and trace mass 43, compound is single bond Hexadecane $\left(\mathrm{C}_{16} \mathrm{H}_{34}\right)$, retention time 18.49 and trace mass 41 , compound is single bond Heptadecane $\left(\mathrm{C}_{17} \mathrm{H}_{36}\right)$, retention time 19.59 and trace mass 57 , compound is single bond Octadecane $\left(\mathrm{C}_{18} \mathrm{H}_{38}\right)$, retention time 20.63 and trace mass 43, compound is single bond Eicosane $\left(\mathrm{C}_{20} \mathrm{H}_{42}\right)$. At the initial phase of the analysis index retention time 21.56 and trace mass 55 , compound is double bond 1-Docosene $\left(\mathrm{C}_{22} \mathrm{H}_{42}\right)$, retention time 22.59 and trace mass 57, compound is single bond Heneicosane $\left(\mathrm{C}_{21} \mathrm{H}_{44}\right)$, retention time 25.25 and trace mass 57 , compound is single bond Tetracosane $\left(\mathrm{C}_{24} \mathrm{H}_{50}\right)$, retention time 26.07 and trace mass 57, compound is single bond Octacosane $\left(\mathrm{C}_{28} \mathrm{H}_{58}\right)$ and ultimately retention time 27.68 and trace mass 57 compound is single bond Heptacosane $\left(\mathrm{C}_{27} \mathrm{H}_{56}\right)$ etc.

Produced fuel boiling point and enthalpy value analys is purpose a DSC (Perkin Elmer) was used shown in fig. 5, 0. Carrier gas was used as Nitrogen $\left(\mathrm{N}_{2}\right)$ at $20 \mathrm{ml}$ per minute. Aluminum pan was used for sample holding and sample used 50 micro liters. Program temperature for DSC was set and ranged from $5^{\circ} \mathrm{C}$ to $400^{\circ} \mathrm{C}$ and ramp at $10^{\circ} \mathrm{C}$ per minutes. Plastic to fuel analysis was by DSC for fuel boiling point measuring. From DSC graph fuel onset temperature is $20.01^{\circ} \mathrm{C}$ and fuel boiling point peak showed $135.32^{\circ} \mathrm{C}$, peak height heat flow Endo up is $17.4933 \mathrm{~mW}$. Peak end temperature is $144.56^{\circ} \mathrm{C}$ and peak area is $9176.852 \mathrm{~mJ}$ and enthalpy value delta $\mathrm{H}$ is $9176.8516 \mathrm{~J} / \mathrm{g}$. Produced fuel went through ASTM test was performed by $3^{\text {rd }}$ party Intertek laboratory New Jersey, USA. Gross BTU - LB (ASTM D240), Gross BTU - gallon calculated (ASTM D240), Freezing Point ${ }^{\circ} \mathrm{C} /{ }^{\circ} \mathrm{F}$ (ASTM D2386), Fuel metal content (ASTM D5708), Sulphur (ASTM D5453), Pour point ${ }^{\circ} \mathrm{C} /{ }^{\circ} \mathrm{F}$ (ASTM D97), Cloud Point ${ }^{\circ} \mathrm{C} /{ }^{\circ} \mathrm{F}$ (ASTM D2500), IBP recovery (ASTM D86), API gravity at $60^{\circ} \mathrm{C}$ (ASTM D4052), Temperature (ASTM D2624), Electrical conductivity (ASTM D2624), ASTM Color (ASTM D1500), Ash @ $775^{\circ} \mathrm{C}$ (ASTM D482), Corrected flash point (ASTM D93) and acid number (ASTM D664). Residue or black coal was analyzed by ICP ASTM D1976 method fo llowed and residue has more metal contain ppm level then raw materials such as Silver, Aluminum, Boron, Barium, Calcium, Chromium, Copper, Iron, Potassium, Lithium, Magnesium, Molybdenum, Sodium, Nickel, Phosphorus, Lead, Antimony, Silicon, Tin, Strontium, Titanium, Thallium, Vanadium and Zinc etc. All metal content present limits residue inside more than waste plastic ppm level. Produce fuel metal content checked by ICP and content found less then $<1 \mathrm{ppm}$ which is negligible for liquid fuel.

\section{Conclusions}

The thermal degradation of polymer waste plastics was performed using muffle furnace through in a stainless steel reactor was shown to be a useful method for the production of potentially valuable hydrocarbons liquid fuel. The experiments discussed in this work show that the use of various ways improves the yield of hydrocarbon fuels and provide better selectivity in the product distributions. Overall, the light of the hydrocarbon products observed were in the gas phase with less than $10 \%$. It is demonstrated that the conversion of post-consumer polymer non coded waste plastic to hydrocarbon fuels was more than $85 \%$ reactor used in this study. Moreover, the production of hydrocarbon as a chemical feedstock is potentially attractive and may offer greater profitability than production of saturated hydrocarbons and aromatics. Thermal degradation resulted in larger amounts of liquid hydrocarbons compared with the degradation over showed the lowest conversion light gas and generated a hydrocarbon product with the broadest carbon range. Greater product selectivity was observed thermal cracking with about liquid fuel $85 \%$ and light gas $9 \%$ was from $1^{\text {st }}$ step liquefaction process and $2^{\text {nd }}$ step condensation process and total conversion rate was $94 \%$ of the product in the $\mathrm{C}_{3}-\mathrm{C}_{28}$ range studied. It is concluded that the use of appropriate reaction conditions without catalyst to a suitable way can give the ability to control product yield and distribution from non-coded waste polymer degradation, potentially leading to a cheaper process with more valuable products.

\section{List of Abbreviations:}

CDS: CDS Analytical, Inc. is a GC/MS Pyroprobe 5000 series manufacturer co mpany

RCI: RCI is a manufacturer of fuel purification technology that is commercially available

KRS-5: (FT-IR component) Diamond plate for FT-IR liquid and solid analysis.

ATR: (FT-IR component) (Attenuated Total Reflectance) is an universal ATR sampling accessory (for analyzing solid samples)

TGA: (Thermogravemetric Analyzer) is an equipment use to determine melt ing point

DSC: (Differential Scanning Calorimeter) is an equipment use to determine boiling point, melting point and free zing point of liquid substances.

ICP: (Induced Couple Plasma) is an equipment used for various metal content analyzing

ASTM: American Standard and Testing Method is an organization that provides chemical characteristics and handling procedure. 


\section{ACKNOWLEDGEMENTS}

The author acknowledges the support of Dr. Karin Kaufman, the founder and sole owner of Natural State Research, Inc. The authors also acknowledge the valuable contributions NSR laboratory team members during the preparation of this manuscript.

\section{REFERENCES}

[1] M. Stelmachowski, 'Energy Conversion and Management', E. Con. and Man, 51: 2016-2024, 2010.

[2] H. Wei-Chiang Mao-Suan, H. Chiung-Fang, C. Chien-Chung, O. Keng-Liang, "Thermochemical conversion of polymer wastes into hydrocarbon fuels over various fluidizing cracking cataly sts', Fuel, 89: 2305-2316, 2010.

[3] G. Scott, Polym. Degrad. Stabil 68: 1, 2000.

[4] K. Fouhy, I. Kim, S. Moore, E. Culp, Chem. Eng. 100: 30, 1993.

[5] S. Shelley, K. Fouhy, S. Moore, Chem. Eng. 99: 30, 1992.
[6] P.L. Beltrame, P. Carniti, G. Audisio, F. Bertini, Polym. Degrad. Stabil. 26: 209, 1989.

[7] G. Manos, K. Vertsonis, A. Garforth, J. Dwyer, in: Proceedings of the 1997 Jubilee Research Event, IChemE p.1193, 1997.

[8] N. Shah, J. Rockwell, G.P. Huffman, Energy Fuel 13: 832, 1999.

[9] I. Nakamura, K. Fujimoto, Catal. Today, 27: 175, 1996.

[10] G. De la Puente, J.M. Arandes, U. Sedram, Ind. Eng. Chem. Res., 36: 4350, 1997.

[11] A.R. Songip, T. Masuda, H. Kuwahara, K. Hashimoto, Appl. Catal. B 2 (2-3): 153, 1993.

[12] W. Ding, J. Laing, L.L. Andersen, Fuel Process. Technol., 51: 47, 1997.

[13] Z. Zhibo, S. Nishio, Y. Moriaka, A. Ueno, H. Ohkita, Y. Tochihara, Y. Mizushima, N. Kakuta, Catal. Today, 29: 303, 1996.

[14] Y. Uemichi, M. Hattori, T. Itoh, J. Nakamura, M. Sugioka, Ind. Eng. Chem. Res., 37: 867, 1998.

[15] H.S. Joo, J.A. Guin, Fuel Process. Technol., 57:25, 1998. 